Muriel Darmon

\title{
Sélectionner, élire, prédire : le recrutement des entrants en classes préparatoires
}

À partir d'une enquête par observation et entretiens portant sur des classes préparatoires scientifiques et économiques du lycée $\mathrm{L}^{*}$, l'article propose une analyse sociologique des procédures et processus d'admission en classe préparatoire, et notamment des catégories de l'entendement professoral qui s'y manifestent. Il évoque dans un premier temps comment elles s'inscrivent dans un contexte politique et professionnel particulier, celui d'une « politisation » de la question des classes préparatoires, qui joue comme contrainte, historiquement située, dans leur énonciation. Il montre ensuite comment elles prennent place dans des luttes de classements entre enseignants (à travers le couple catégoriel central du «travail » et du «niveau ») et comment elles mêlent évaluation et prédiction des dispositions étudiantes à travers la quête de «l'énergie scolaire » des candidats.

Des étudiants «sélectionnés »: dans le discours public sur les classes préparatoires, le participe passé efface le verbe. Par cette expression sont en effet fréquemment rappelés, sous forme concentrée, à la fois les qualités et les privilèges de la population scolaire particulière des classes préparatoires. Mais avant de fonctionner comme la marque d'un statut, la sélection est une opération.

Son analyse dans le cas des classes préparatoires est d'autant plus nécessaire que la sociologie a également privilégié le produit au détriment du processus. Ce que l'on connaît de la sélection des entrants en classes préparatoires, c'est ce que l'on sait, année après année, de la composition des classes à laquelle elle aboutit : on mesure notamment leur recrutement social toujours biaisé en faveur des enfants des classes supérieures ${ }^{1}$. En ce qui concerne la sélection des classes préparatoires, objet sociologique et problème social sont donc tous deux

1 Voir par exemple (Pons, 2007) et les autres Note d'information de la DEPP consacrées aux étudiants de classes préparatoires. 
construits ou focalisés autour de la question de la contribution du système des classes préparatoires à la reproduction sociale et de la mesure critique de son élitisme. D'où l'intérêt de ne pas s'y limiter, et de décentrer le regard du résultat à l'opération qui préside au recrutement des élèves. On avancera en effet ici que l'analyse sociologique de ce recrutement ne s'épuise pas dans la mise en lumière statistique de ses biais, de même que l'étude des classes préparatoires ne se réduit pas à celle de leurs effets sur les inégalités scolaires et sociales.

Le matériau central livré par l'observation des opérations de recrutement, ce sont les catégories de l'entendement professoral qui s'y illustrent et qui les guident. Ces « schèmes de perception, d'appréciation et d'action », «acquis par la pratique et mis en œuvre à l'état pratique ", s'inscrivent dans des taxinomies et des formes scolaires de classification que leur observation permet d'approcher (Bourdieu, Saint Martin, 1975) ${ }^{2}$. Elles sont au centre de cet article, mais celui-ci ne vise pas pour autant, contrairement à la mission originelle attribuée au concept, à établir une relation entre ces catégories et les origines sociales des élèves au sujet desquels elles sont émises —on ne dispose pas des données nécessaires à ce type d'objectivation. Mais l'intérêt de l'analyse de ces systèmes de catégories ne s'y réduit heureusement pas, comme on tentera de le montrer en saisissant ces catégories en acte, comme une dimension d'un travail professoral en train de se faire. De plus, on utilisera la manière dont l'examen de ces catégories permet de s'émanciper d'une approche universaliste et statique des «valeurs » enseignantes ou des « représentations » professorales prises comme un tout, invariant (dans le temps) et invariable (selon les individus) : « [Le fait qu'elles soient structurées] ne signifie pas qu'elles soient justiciables d'une analyse strictement interne («structurale » (...) ou autre) qui, en les arrachant artificiellement à leurs conditions de

2 Voir aussi l'intervention de P. Bourdieu in (Cardi, Plantier, 1993) où elles sont définies comme des «transcendantaux, c'est-à-dire des formes a priori de classification que les sujets sociaux mettent en œuvre dans leur perception, dans leur évaluation du monde et dans leur pratique ", mais qui n'en sont pas moins " produites dans et par un certain type de conditions historiques », p. 197-198. 
production et d'utilisation, s'interdit d'en comprendre les fonctions sociales » (Bourdieu, Saint Martin, 1975). C'est dans cet esprit — qu'on pourrait dire sociologique davantage qu'anthropologique - que l'on se propose de mettre ici en lumière certains principes de variations auxquels ces catégories obéissent, et donc de montrer qu'elles sont soumises à diverses contraintes non scolaires et à divers usages scolaires. On verra ainsi dans un premier temps comment elles s'inscrivent dans un contexte particulier qui joue comme contrainte, historiquement située, dans leur production et énonciation. On se concentrera ensuite sur ces catégories elles-mêmes et les taxinomies qu'elles révèlent, en montrant comment elles prennent place dans des luttes de classements entre enseignants, voire dans un espace social des jugements scolaires, et comment elles mêlent évaluation et prédiction des dispositions étudiantes. Par rapport à une vision des catégories de l'entendement professoral comme partagées et consensuelles, et comme purement évaluatrices, on cherche donc à mettre en lumière les variations dans lesquelles elles s'inscrivent et la diversité de leurs usages sociaux.

\section{Encadré 1 : Enquête et matériaux}

Les matériaux utilisés ont été recueillis au cours d'une enquête ethnographique menée pendant deux ans sur les classes préparatoires scientifiques et commerciales du lycée L*. À partir des premiers jours de la rentrée de quatre classes de première année (deux classes scientifiques, deux classes économiques) jusqu'aux résultats des concours de ces mêmes élèves en fin de deuxième année, une centaine d'entretiens (94) ont été réalisés avec les mêmes élèves à différents moments de ces deux années, ainsi qu'avec des enseignants (12) et des administrateurs du lycée (2). Ces entretiens ont été inscrits dans le cadre d'une présence longue au lycée et articulés à des séquences d'observation, non participante et à découvert (une centaine d'heures) en classe, en colle, dans les conseils de classe, des réunions diverses et des moments forts, formels ou informels, de ces classes. 
On utilise plus spécifiquement pour cet article les extraits du journal de terrain consacrés à l'observation de deux réunions de commissions d'admission en classes préparatoire et les entretiens menés avec les enseignants, le proviseur et un CPE du lycée.

La procédure de sélection des nouveaux entrants utilisée au moment de l'enquête, en vigueur depuis 2003, est informatisée (les candidats s'inscrivent sur un site internet et leurs dossiers circulent à la fois sous forme papier et sous forme informatisée). Les élèves de terminale candidats peuvent émettre, et classer par ordre de préférence, jusqu'à 12 voeux (le point de vue, les stratégies et l'univers des possibles des candidats n'étant pas analysés dans l'article). Chaque commission d'admission (composée en théorie du proviseur, du proviseuradjoint chargé des classes préparatoires, et des enseignants de la classe pour laquelle les demandes sont faites) doit donc se prononcer sur les candidatures qui lui parviennent. Les commissions d'admission observées (l'une en classe scientifique, l'autre en classe commerciale) ont duré respectivement une journée et une grosse demi-journée, et ont réuni les enseignants de première et deuxième année de chacune des classes (ainsi qu'à de rares moments le proviseur-adjoint), avec une présence quantitativement plus longue, et un poids plus évident dans les décisions prises, des enseignants des «matières importantes » dans chacune.

Le lycée enquêté fait partie des «bons » lycées de province, et occupe de ce fait une place qu'on pourrait dire «intermédiaire» dans la hiérarchie symbolique des classes préparatoires entre les «grands lycées parisiens » (connus nationalement, et reconnus pour «placer» leurs élèves dans les plus prestigieuses des grandes écoles) et les «petites prépas des petits lycées de province » (dont les élèves n'accèdent qu'exceptionnellement aux plus grandes des grandes écoles). A ceci s'ajoute une différence sensible de recrutement et d'univers des possibles préparatoires, qui distingue les prépas scientifiques des prépas économiques enquêtées à nouveau en termes de hiérarchies symboliques tant locales que 
nationales. Les prépas scientifiques du lycée $\mathrm{L}^{*}$ y sont vues comme «les meilleures prépas du lycée », et des élèves des classes dites «étoilées » rentrent tous les ans dans les «meilleures écoles ». Les prépas économiques sont elles davantage perçues et décrites dans le lycée comme «moins bonnes » - la qualification signifiant à nouveau que c'est la réussite des élèves dans les concours les plus prestigieux, plus rare, qui sert de critère.

\section{Une opération de sélection particulière}

Les observations effectuées au lycée $\mathrm{L}^{*}$ permettent de faire apparaître le contexte professionnel et politique particulier dans lequel s'effectue aujourd'hui la sélection des entrants en prépa, contexte qui encadre, voire contraint la production et l'énonciation des catégories de l'entendement professoral. Cette première partie est destinée à le mettre en lumière en cherchant à caractériser l'opération de sélection particulière à l'étude. Il s'agit d'une sélection (1) «sur dossier», (2) largement informatisée, qui s'effectue (3) sous contrainte administrative et politique.

\subsection{Une sélection sur dossiers}

Les deux commissions d'admission auxquelles j'ai assisté ont réuni, pendant une journée ou une demi-journée, les enseignants de première et deuxième année de chaque classe préparatoire dans une salle où sont stockés pour l'occasion les dossiers envoyés par les lycéens ayant indiqué dans leurs vœux la classe du lycée L*. Ce sont ces dossiers qui, sous forme papier ou informatisée, constituent le matériau sur lequel travaille la commission.

Le fait que la sélection ne se fasse pas, par exemple, à partir d'une interaction de face-àface avec le postulant, spécifie déjà le type de procédure engagée même si un tel mode de sélection est évidemment fort répandu dans l'institution scolaire ou le marché du travail. Cependant, par différence avec d'autres modes de sélection sur documents effectués par 
l'institution scolaire, celui-ci s'élabore non à partir d'un matériau «brut » à évaluer, comme dans le cas des devoirs écrits des examens et des concours, mais à partir du concentré d'évaluations constitué par le dossier scolaire de l'élève. Il s'agit donc clairement, pour les commissions, d'élaborer un jugement scolaire à partir d'autres jugements scolaires, et donc d'effectuer une évaluation de second degré.

Cela est particulièrement visible, et explicite, lorsque les enseignants membres de la commission mettent en scène leur capacité à « décoder » les appréciations de leurs collègues de lycée, à rétablir les jugements euphémisés ou à identifier les non-dits véhiculés par des catégories partagées de l'entendement professoral. Ces dernières fonctionnent alors comme une langue d'initiés, permettant de se parler par dossier interposé, comme le montrent ces deux exemples, tirés de l'examen des dossiers par une commission de classes préparatoires commerciales :

Un enseignant lit une première appréciation d'un dossier : "Paul est la voix de la terminale S3... », «Ouh là, intervient immédiatement un autre, la voix c'est pas le cerveau! » L'enseignant continue: "Le proviseur met 'sympathique, tire son épingle du jeu'....», appréciation qui est accueillie avec des rires entendus, un des membres du comité explicite: «Bon, OK, il sera bon en entretien lui, c'est un petit malin, mais il y aura quand même des problèmes à l'écrit... ».

Sur un autre dossier, un premier enseignant: «En maths, le prof dit qu'il est “'curieux', c'est sympathique chez un élève, mais on sait que ça veut pas dire 'bon'”! "; «En plus, renchérit un deuxième enseignant de la commission, d'après le proviseur il a la "capacité à suivre des cours de classe préparatoire", ce qui veut bien dire qu'il n'a pas forcément la “'capacité de réussir en devoir surveillé”! » [Journal de terrain (JT)].

Une partie du travail d'évaluation consiste ainsi en un décryptage des évaluations antérieures telles qu'elles sont transmises par le dossier scolaire, décryptage qui est rendu plus 
facile par le partage des catégories d'évaluation entre enseignants rédacteurs du dossier et enseignants membres de la commission.

\subsection{Une sélection sur fichier}

Une deuxième caractéristique importante du processus de sélection à l'œuvre tient à sa dimension pour une large part informatisée. Si les dossiers papier des candidats sont d'emblée présents dans la salle, tout le monde est, dans un premier temps, soit réuni autour de l'ordinateur et de son tableau Excel, dans lequel les dossiers des candidats ont été entrés et classés, soit penché sur la version papier du tableau et du classement informatiques. C'est donc le classement réalisé par le logiciel qui joue le rôle de base de départ : si la sélection n'est pas déjà faite, et si «le» classement final ne préexiste pas à la réunion de la commission, le travail de cette dernière s'élabore cependant à partir d' « un » classement réalisé par l'outil informatique.

Ce classement n'est cependant ni aveugle ni purement technique, dans la mesure où les données qu'il a prises en charge et le type de traitement qu'il leur a fait subir sont eux-mêmes gouvernés par les modes scolaires et professoraux d'appréciation de la réalité : le logiciel fonctionne comme un résumé ou une cristallisation informatiques des catégories de l'entendement professoral. Le choix des indicateurs retenus est ainsi cohérent avec les caractéristiques pertinentes de l'élève dans le système scolaire français ${ }^{3}$ : indication de la provenance des candidats, à la fois géographique (lycée d'origine, ville, département) et scolaire (type de lycée, de section en terminale, évaluation du niveau de la classe dont est issu chaque candidat sur une échelle de 1 à 4), avis du chef d'établissement (également sur une

${ }^{3}$ L'évidence de cette définition de l'élève par ses caractéristiques strictement scolaires s'efface quand on compare cette procédure avec celle utilisées dans les universités prestigieuses aux ÉtatsUnis, où le processus de sélection met en jeu des critères explicitement non académiques tels que les activités extra-curriculaires, la pratique sportive, le fait d'être un enfant d'ancien élève, et l'importance explicitement donnée à la «noblesse de caractère », la " personnalité » ou le « leadership » (Karabel, 2006) qui sont autant de « vertus mesurables» par les responsables des admissions (Stevens, 2007). 
échelle de 1 à 4), moyennes et classements de l'élève selon les matières pour la classe de terminale et pour certaines matières de la classe de première, ou encore notes aux épreuves anticipées du baccalauréat.

De plus, la construction du niveau de l'élève effectuée par le logiciel est adaptée à la classe préparatoire concernée, au moyen d'un paramétrage : les différentes moyennes de l'élève sont affectées de coefficients variables selon les disciplines, et certaines formules spécifiques de classement peuvent être introduites. Ainsi, en ce qui concerne la commission de la classe préparatoire scientifique, des formules éliminatoires sont prévues et «extraient » du classement de départ les candidats ayant des moyennes en mathématiques, en mathématiques + physique-chimie, en français ou en LV1 inférieures à une moyenne fixée.

L'outil informatique fonctionne comme une ressource indispensable dans un processus qui s'effectue désormais à une échelle rendant très difficile le traitement au cas par cas. Mais il fonctionne aussi dans une large mesure comme une boîte noire pour la commission, qui travaille sur le classement qu'il produit sans avoir accès à ses principes de fonctionnement et sans pouvoir modifier les critères sur lesquels il repose. Il est donc en fait partie intégrante d'un dispositif de contraintes étendu dans lequel évolue, et évalue, la commission.

\subsection{Une sélection contrainte administrativement}

Parmi ces contraintes, le poids des décisions et injonctions administratives est particulièrement visible. Tout d'abord, la commission ne se prononce pas, légalement, sur les décisions d'admission: elle procède à un classement et transmet un avis au chef d'établissement en ce sens. Mais l'administration du lycée est loin de n'intervenir qu'en bout de course. Dans les deux commissions observées, les figures du proviseur et de son adjoint chargé des classes préparatoires sont très présentes et pèsent sur les décisions qui y sont prises. Dans l'une des commissions, l'enseignant qui organise les débats commence ainsi en mentionnant la barre — c'est-à-dire la moyenne en-dessous de laquelle tous les dossiers sont 
refusés - souhaitée par « le patron », et qui sera bien in fine la barre fixée par la commission. Dans une autre, un enseignant intervient au début des débats pour demander à ce qu'on « relève le niveau » en modifiant les critères éliminatoires dans le logiciel (ce qui pourrait entraîner des classes moins chargées et composées d'élèves aux meilleurs dossiers), mais malgré la discussion qui s'ensuit il apparaît très vite qu'il serait «trop compliqué » de les changer, qu'il faudrait «demander au proviseur adjoint», et que ces critères constituent en fait des données non modifiables (et fixées ex ante) pour la commission. Le cadrage administratif des opérations d'évaluation et de sélection scolaires est donc ici tout à fait explicite.

La question du « remplissage » des classes (qui dans le cas des classes préparatoires se combine avec celle du maintien de l'effectif tout au long de l'année) est plus généralement un enjeu potentiel de tiraillement voire de luttes entre les segments administratif et pédagogique de l'institution scolaire étudiée. L'enseignant évoqué ci-dessus, qui a essayé d'obtenir la modification des critères d'exclusion, explique ainsi à deux collègues, sur le pas de la porte et à la fin de la commission, ce qu'il désigne comme le «contexte » de cette dernière : [Les enseignants principaux de la classe] se sont fait « remonter les bretelles » par le proviseur adjoint suite au départ de deux élèves en cours d'année. Le PA leur reproche de les avoir laissé, voire "fait » partir, et leur a dit que l'administration regardait ça de très très près, qu'un élève de prépa, ça coûtait horriblement cher, et qu'à partir de maintenant, il allait vraiment falloir que personne ne parte au cours de l'année, et que tout le monde passe en deuxième année, vu le coût d'un élève de prépa en première année, il fallait que ce soit rentable...[JT].

Or l'efficacité et le pouvoir de conviction de la thématique du remplissage — y compris auprès des enseignants eux-mêmes, qui ont pourtant à y perdre en termes de conditions de travail — proviennent de la manière dont elle combine un réquisit gestionnaire avec la teneur 
plus sociale d'une vision des classes préparatoires comme « ouvertes », donnant leur chance à davantage d'élèves et implicitement opposées au schème de la classe préparatoire élitiste « d'avant», comme le montre par exemple cet échange en commission : Lors du débat sur la fixation de la «barre », l'enseignant responsable de la réunion pousse plutôt à descendre dans la liste et à déclarer admissibles davantage de candidats : "L'idée, c'est de faire le plein [des classes], d'aller à 48 [élèves par classe]. Faire le plein, c'est la politique officielle... ». Un autre enseignant, acquiesçant : "Et puis c'est bien de donner leur chance à un maximum de gens, qui peuvent être des gens méritants. Même pour une question de gestion des deniers publics, l'attitude responsable c'est de continuer [à descendre dans le classement $] \gg[\mathrm{JT}]$.

La question du «coût » d'un élève de classes préparatoires, qui transparaît dans les extraits ci-dessus, concentre elle aussi ces deux dimensions, gestionnaire et sociale, et c'est sans doute précisément pour cette raison qu'elle s'est imposée si visiblement comme un problème social central concernant les classes préparatoires. Le proviseur du lycée accueille d'ailleurs chaque année les entrants en l'évoquant: «Les collègues se sont étonnés que je mentionne la question économique [lors de la réunion d'accueil de rentrée des élèves de prépa], mais c'est un problème public maintenant, et les élèves ça les rassure d'entendre que, si on prend bien les choses, ils ne coûtent pas plus cher qu'un élève de la fac » (Proviseur du lycée $L^{*}$ à l'enquêtrice, à la sortie d'une réunion de rentrée). On a vu plus haut comment la question du «coût » constituait également un rappel des enseignants à l'ordre administratif. Elle figure de plus dans certains entretiens avec eux comme celle par laquelle le droit de regard public sur leurs pratiques professionnelles se fait le plus sentir :

- [Lors d'un entretien non enregistré en salle des profs avec un enseignant de sciences en prépa scientifique :] Il me détaille «les attaques contre la prépa » il y a des gens qui disent qu'elles devraient être supprimées car elles coûtent trop cher et ne profitent qu'à une 
élite. Les profs ont eu une réunion avec l'Inspection, qui leur a dit "vous êtes dans le collimateur », «dans la ligne de mire», «on attend que vous fassiez un seul faux pas pour vous supprimer $»[\mathrm{JT}]$.

- «C'est le gros reproche qu'on nous fait essentiellement, dans les débats actuels, que c'est trop cher par élève... (...) Mais c'est vrai que ça c'est une des choses qui nous embête en ce moment, le débat actuel là-dessus, parce qu'on ne sait pas du tout ce que ça va donner, on emploie des arguments... complètement délirants pour nous, dans les médias, par des universitaires entre autres, des journalistes, et ça c'est vraiment, c'est très difficile je trouve.

Enquêtrice : Sur le côté : "C'est pour les privilégiés », c'est ça?

- Voilà, oui c'est ça, alors que le taux de boursiers est quand même pas si négligeable que ça, et en particulier chez nous il est plus élevé que la moyenne... On a toujours cette tendance à dire que le prix par élève, enfin le coût par élève est peut-être élevé mais tous arrivent au bout, alors que finalement rien qu'en ramenant au nombre d'élèves qui réussissent leur Deug... »(Enseignant sciences, prépa scientifique).

La question du « coût » d'un élève de classes préparatoires, en fait de son coût relatif à celui d'un étudiant de l'université, s'inscrit dans le contexte plus large de ce que l'on pourrait qualifier de «politisation » (Lagroye, 1997) de la question des classes préparatoires, qui pèse sur l'encadrement administratif des procédures de sélection. Cette politisation s'illustre par la médiatisation des prépas comme question politique ou de politique scolaire depuis le milieu des années 1990 (Allouch, Van Zanten, 2008) (Pasquali, 2010), ainsi que par la perception par les enseignants d'un cadrage officiel croissant des décisions scolaires. Dans ce contexte, l'institution préparatoire ne doit plus se voir comme un monde scolaire autonome mais doit répondre à des demandes « sociales » (par exemple de mixité sociale, d'augmentation de la proportion des boursiers, mais également de la proportion de filles dans les sections scientifiques) et «économiques » (fournir aux grandes écoles, puis au marché du travail, 
suffisamment d'élèves, disposant d'une formation adéquate, plus «concrète »), thèmes que l'on a donc vu apparaître dans les discussions des commissions.

Le travail de sélection et d'évaluation qui est opéré pendant ces commissions est donc un travail encadré, voire contraint, par l'usage de l'outil informatique ou les directives administratives et politiques. Le tout en fait une opération de sélection particulière, qui, si elle s'apparente a priori à l'élaboration d'un simple classement, combine en fait tout un ensemble de procédures d'élection, d'élimination, de qualification, d'acceptations ou de refus sur lesquelles, une fois leur contexte rappelé, il est maintenant possible de se pencher, en se concentrant sur les catégories qui sont mobilisées à l'occasion de ces opérations et qui gouvernent ou justifient les décisions qui y sont prises.

\section{2. Élire, éliminer : la « machine simple » et la « machine}

\section{cognitive »}

À ne considérer, au niveau statistique, que l'origine sociale des élèves acceptés en classes préparatoires, on pourrait être conduit à ne voir dans la procédure de sélection de ces élèves que « le schéma d'une machine simple qui, recevant des produits hiérarchisés selon un classement social implicite, restitue des produits hiérarchisés selon un classement scolaire explicite, mais très proche en réalité du classement initial »- et le logiciel de classement des dossiers joue d'ailleurs très bien ce rôle de «machine simple ». Mais ce serait là, tout comme dans le cas de la notation des copies de philosophie étudiée par P. Bourdieu, «laisser échapper l'effet propre de cette étrange machine cognitive qui opère toute une série d'opérations de connaissance et d'évaluation » (Bourdieu, 1989) : c'est-à-dire l'effet des catégories de l'entendement professoral. 
S'interroger sur le rôle joué par ces catégories, c'est tout d'abord s'interroger sur les contours concrets de leur domaine d'action. Si la discussion au «cas par cas » des dossiers des élèves et l'examen de leur «personne » scolaire jouent comme normes des travaux de la commission d'évaluation (les textes qui présentent le travail des commissions sont très discrets sur l'emploi des logiciels), l'examen de la forme prise par les commissions montre que la sélection ne se fait pas d'abord, et ne se fait pas en totalité, selon ce modèle : 1'espace d'intervention de la « machine cognitive » est un espace restreint.

Certes, les catégories de l'entendement professoral interviennent en un sens dans l'ensemble de la procédure, parce qu'elles existent sous formes institutionnalisées et objectivées dans la production des dossiers et des notes scolaires des élèves en première et terminale, et dans la conception même du logiciel de classement, ainsi que dans son paramétrage. Mais l'exemple des commissions observées montre que «la discussion sur les dossiers », c'est-à-dire la manifestation sous forme pratique, explicite et débattue de ces catégories, est cantonnée à un espace particulier des profils scolaires, à savoir la frange des dossiers «moyens », «énorme marais» (pour reprendre une expression utilisée par un enseignant) dont les caractéristiques objectives sont variables de classe en classe mais qui a comme point commun d'être défini par une double exclusion, celle des premiers dossiers qu'on accepte automatiquement (et a priori dans l'ordre du classement opéré par le logiciel), et celle des dossiers situés en dessous de la «barre » d'acceptation fixée.

En fait, les premières discussions qui ont lieu dans les deux commissions à propos de ces deux exclusions peuvent être lues comme ayant pour but l'établissement d'une division du travail entre machine simple et machine cognitive. Cette répartition des tâches a pour effet de concentrer le travail de la machine cognitive sur les dossiers «du milieu » du classement, frange qui est précisément, du fait de la position intermédiaire du lycée, celle où les classes vont recruter majoritairement leurs élèves. Sans que cela soit mentionné ni même forcément 
conscient lors des réunions des commissions, la discussion au cas par cas est certes limitée, mais elle porte donc sur le groupe le plus probable des candidats.

Ce premier temps de la discussion aboutit donc tout à la fois à une sélection (la reprise par la commission du classement du logiciel pour les premiers du classement), à une exclusion (le refus des dossiers dont la moyenne générale calculée par le logiciel est inférieure à la «barre » discutée), et à la définition d'un « lieu » intermédiaire pour lequel se met en branle la «machine cognitive » des catégories de l'entendement professoral.

\subsection{Les catégories de l'entendement professoral comme outil d'évaluation}

Pour les étudier, on peut partir du double travail que mène, sur les dossiers «moyens », la commission d'admission en classe préparatoire scientifique observée: un travail de sauvetage, consistant à regarder les dossiers ayant une bonne moyenne mais ayant été exclus du classement par l'un des critères éliminatoires, un travail d'élimination qui repère les dossiers ayant les moyennes les plus faibles mais néanmoins classés, dans l'idée de les sortir éventuellement du classement. Dans les deux cas, les enseignants se penchent donc sur les dossiers scolaires papier des candidats, lisent tout haut les appréciations rédigées par les enseignants de lycée, les décryptent et y ajoutent leurs propres commentaires. Les catégories de l'entendement professoral sont donc ici à la fois le matériau sur lequel vont s'appuyer les enseignants de la commission (et qui joue, on l'a vu plus haut, comme langage codé, d'enseignant à enseignant), et l'outil cognitif et argumentatif qu'ils vont personnellement mettre en œuvre.

\section{« Matières importantes » et « petites matières »}

En ce qui concerne le travail de sauvetage, il fait immédiatement intervenir la hiérarchie des disciplines : toutes les notes ne se valent pas. Les coefficients assignés à chaque moyenne par le logiciel informatique, qui objectivent cette hiérarchie, ne l'objectivent pas une fois pour 
toutes, et ils sont redoublés par les effets de la discussion sur les cas tangents. Ce travail de sauvetage est défini au départ par un enseignant comme une manière de « sauver ceux qui ont péché sur un des critères ", mais il s'agit en fait surtout de repêcher des élèves ayant de bonnes moyennes scientifiques mais «coulés » par une note en français ou en anglais. Un certain nombre de candidats, mauvais en lettres, mais bons en sciences, sont ainsi « sauvés ». Comme lors de toutes les observations (en conseil de classe, en commission, en réunion...) que j'ai pu mener par ailleurs dans les classes scientifiques, l'humour est un mode habituel de rappel de ces hiérarchies entre matières. Chacun joue en quelque sorte son rôle avec bonne humeur, les enseignants des «matières importantes» rappelant et atténuant les hiérarchies existantes, les enseignants des «petites matières » les acceptant et y résistant :

À un moment, un élève ayant une note particulièrement basse en langues est « rattrapé » : " on le cache à X [l'enseignant de langues présent, qui discute sur un autre cas et n'entend pas] !! », dit un prof de science en faisant mine de chuchoter ; les enseignants de science présents rigolent, l'un d'eux ajoute : «oui, mais bon, en faisant ça, c'est vrai qu'on est en train de pourrir les classes de langues!».

A midi, X se lève pour partir, et lance en riant à son collègue de science : "Maintenant que je m'en vais tu vas pouvoir prendre tous les nuls en langues!».

\section{Esprit de sérieux et docilité}

À cet ajustement à la spécificité de la classe préparatoire concernée s'ajoute la recherche, par les enseignants, d'indices concernant «l'attitude» des candidats. L'enquête confirme en effet que la sélection des candidats se fait « en fonction de leurs dispositions à l'égard de l'école, c'est-à-dire de leur docilité, au moins autant qu'en fonction de leurs aptitudes scolaires » (Bourdieu, 1989).

Les indices clairs d'un manque de sérieux ou de docilité par rapport à l'École ont ainsi systématiquement pour effet d'exclure le repêchage du candidat. Des candidats dont les 
appréciations comportent un «avis réservé du chef d'établissement» («On devrait en prendre aucun, [dans une autre classe, reconnue comme "la meilleure du lycée »] ils n'en prennent jamais »), la mention d'《absence aux devoirs, bavardage » (« on le prend pas !!! ») ou encore des signes de distance face aux normes ou réquisits scolaires ( « il s’y est mis un peu tard», «la matière ne semble pas l'intéresser »), sont ainsi immédiatement éliminés : «Dissipation, manque de sérieux dans le travail », lit un enseignant. "Quand on voit ça, c'est réglé !! » [élève exclu du classement]. Même les notes dans les «petites matières », si elles en viennent à être interprétées non comme une défaillance scolaire mais comme une mauvaise disposition à l'égard de l'école, en deviennent importantes : "Absence totale de travail en philo »... lit, d'un ton significatif, un enseignant de science. "Oui, mais bon, en philo on s'en fiche un peu non? » "Non! C'est un état d'esprit... », «ça veut dire aussi qu'il est pas prêt à tout pour avoir un bon bac » [élève exclu du classement].

Symétriquement, des candidats peuvent être sauvés presque immédiatement par des signes de bonnes dispositions :

"'Élève vif et attentif", ah, c'est bien... » [Élève « repêché »].

[Discussion entre enseignants pendant la commission : ] "S'il a 15 partout et 10 en maths, et qu'il est sérieux, il arrivera à faire quelque chose ici », "Et puis les profs se sont engagés [dans leurs appréciations, longues et très encourageantes], il faut le prendre!", «Oui, même si c'est clairement un littéraire... »[Élève accepté].

La recherche de ces signes de docilité scolaire s'illustre donc tant dans le travail de sauvetage que dans celui d'élimination.

\section{Être « sérieux » sans être « au taquet »}

Elle coexiste cependant avec le caractère ambigu du «sérieux» scolaire dans les catégories de l'entendement professoral. Le « labeur », le «taquet», sont les figures noires du sérieux scolaire, les revers de la médaille toujours susceptibles de contaminer leur endroit. Les 
enseignants sont de plus conscients de la réversibilité des appréciations professorales du sérieux et ils les décryptent tout à fait tranquillement sous la plume de leurs collègues :

- Un enseignant lit tout haut : «Se donne beaucoup de peine... » Eclat de rire général : «c'est pas très gentil comme appréciation! » [Élève refusée].

•[Dans un dossier :] "'Sérieuse et appliquée..." "Ça, c'est une laborieuse !! Elle ne va rien comprendre, là ce que met le prof c'est qu'elle fait du hors-sujet en maths en terminale! Ça veut vraiment dire qu'elle comprend rien de rien, ça va être une catastrophe en prépa, elle va rien comprendre » [élève refusée].

- L'enseignant lit l'appréciation en rendant, par un ton lent et pénétré, l'idée d'un travail herculéen: "beaucoup de sérieux, des difficultés surmontées grâce aux efforts entrepris » : [Voix normale :] ça rame, ça rame! Elle est au taquet, c'est une laborieuse!» [élève refusée].

- «'A besoin de temps pour assimiler'... Ça, en prépa, ça pardonne pas! » [élève refusée].

Si cette disqualification de la soumission excessive aux normes scolaires a été analysée par Bourdieu et Passeron comme manifestant la perception, par les enseignants, de dispositions de classe éloignées du modèle dominant de l'aisance (Bourdieu, Passeron, 1985), on peut noter que, dans tous les exemples recueillis ici, les laborieux sont des laborieuses. Les catégories professorales apparaissent en effet comme fortement marquées par les dimensions de genre ${ }^{4}$. Pendant la commission elle-même, l'idée selon laquelle ces catégories du «trop sérieux » s'appliquent en fait plus fréquemment aux «trop sérieuses » est d'ailleurs presque

4 Pour une critique rétrospective de la prise en compte insuffisante des différences de sexe, et du caractère presque exclusivement masculin de l'élite scolaire dans La Noblesse d'État, voir (Saint Martin, 2008). Il faut cependant noter qu'en ce qui concerne les catégories de l'entendement professoral, le problème est en un sens inverse puisque, vu le matériau principalement utilisé (les appréciations portées par un professeur de philosophie sur ses élèves dans une khâgne féminine) on peut se poser la question de l'effet du caractère exclusivement féminin des élèves sur la structure et l'usage des catégories, voire du croisement qui s'opère en l'espèce entre violence symbolique scolaire et effets de la domination masculine. 
explicite, comme le montre cet échange entre un enseignant et une enseignante à propos d'un dossier défini comme « sérieux sérieux sérieux » :

«- [Enseignante] Et puis, c'est une fille, bien sûr!

- [Enseignant] Oh, on peut la prendre, va, de toute façon sérieuse comme ça elle ne nous dérangera pas...

- [Enseignante] Oui, les filles ne nous dérangent pas, sauf quand elles bavardent ».

Lorsqu'ils identifient ces cas de sérieux excessif, les enseignants font deux choses : ils évaluent des difficultés potentielles à suivre en classe préparatoire ou à y réussir, et en ce sens le fait d'être déjà jugé, ou jugée, «trop scolaire» en terminale présage sans doute effectivement mal de la classe préparatoire. Mais, ce faisant, ils privilégient aussi un certain type de problèmes potentiels par rapport à d'autres, et sont ainsi conduits à renforcer, du fait du caractère sexué de cette catégorie, les effets des modes d'exclusion et d'auto-exclusion bien documentés par ailleurs des filles par rapport aux filières scientifiques (C. Marry, 2000) (C. Marry, 2004). Il semble ainsi fort probable qu'à résultats égaux, une fille « sérieuse sérieuse sérieuse » et un garçon qui n'est pas codé comme tel ne soient pas évalués de la même manière lors des processus de sélection.

Plus généralement, les enseignants peuvent être conscients de la violence de ce retournement des catégories scolaires, qui fait « exclure » du classement des élèves appliqués mais «laborieux ». Cette violence reste toutefois pensable et exerçable par eux car elle n'est pas considérée comme gratuite, mais bien comme le moyen d'éviter d'infliger une violence jugée supérieure à celle exercée lors de la commission : à savoir la violence que constitueraient le rythme et le niveau de la classe préparatoire pour des élèves qui n'y seraient pas «à leur place». Le «ça va être une catastrophe » signifie donc aussi « une catastrophe pour elle », et manifeste une attitude de préservation de l'élève. La formule ne s'y réduit pas cependant. Dans la mesure où les exclusions ou même les départs volontaires sont 
administrativement et politiquement découragés, c'est aussi précisément le niveau et le rythme de la classe qui sont préservés par la non-admission de ces élèves-là : il s'agit de disqualifier ex ante faute de pouvoir éliminer ex post, ce qui rend plus décisif l'aspect éliminatoire de la sélection.

\section{2 Les luttes de classement internes à la machine cognitive}

On a pour l'instant considéré dans ce texte les catégories de l'entendement professoral comme des catégories communes et consensuelles. C'est ainsi qu'elles apparaissent notamment lorsqu'on examine leur mobilisation dans les appréciations portées par un même enseignant sur des copies ou des élèves, comme c'est le cas pour le matériau tout à la fois «banal» et « exceptionnel » sur lequel se fondent les analyses de P. Bourdieu et M. de Saint Martin (les notes et appréciations attribuées pendant 4 ans par un professeur de philosophie à ses élèves khâgneuses) (Bourdieu, Saint Martin, 1975) (Bourdieu, 1989). Le fait d'observer les catégories à l'œuvre lors de commissions entraîne un certain nombre de conséquences : on est méthodologiquement conduit à les considérer davantage comme collectives, débattues et variables selon les enseignants. La facilité à décoder les appréciations des collègues, et à en tirer des conclusions communes, qu'on a vu apparaître ci-dessus et qui témoigne d'un consensus, ou en tout cas d'une communicabilité des avis, n'empêche pas que ces catégories de l'entendement professoral évoluent elles aussi dans un espace social, et donnent donc lieu à des débats et des désaccords 5 .

Peut-on dégager certains principes de variations auxquels elles seraient soumises ? Les disciplines enseignées interviennent évidemment : on vu ci-dessus la réversibilité de certaines catégories selon qu'elles émanent d'un enseignant de science ou de lettres - le terme de

${ }^{5}$ L'idée selon laquelle les catégories de l'entendement professoral varient selon les positions objectives des enseignants et leurs pratiques pédagogiques se trouve également in (Merle, 1998, p. 29). 
« littéraire », par exemple, dans la formule «c'est un littéraire », peut être affecté de connotations positives ou négatives selon le locuteur qui l'énonce.

De même, la spécificité de chaque filière conduit à ce que les deux commissions d'admission observées ne s'accordent pas dans tous leurs jugements. La commission en classes préparatoires commerciale porte ainsi la marque de sa relation, même lointaine, à la « réalité managériale » (Abraham, 2007). Par exemple, le capital international accumulé par le fait d'avoir passé une année à l'étranger est très valorisé dans les dossiers examinés — sans qu'il puisse pour autant compenser une situation scolaire délicate. Ou encore, les élèves d'ores et déjà perçus comme des «petits malins», des «tchatcheurs », reçoivent des jugements mitigés, voire positifs chez certains enseignants (et non uniquement négatifs, contrairement à ce qu'exigerait la valorisation classique de l'academica mediocritas comme marque de la bonne distance à l'institution scolaire ${ }^{6}$ ).

Mais la discipline enseignée, la section et les intérêts « catégoriels » qu'elles entraînent sont loin d'être l'unique matrice de ces variations intra-professorales, comme le montre cette discussion animée entre enseignants sur la question du «sérieux » scolaire et le désaccord qu'elle met au jour :

[Commission classe scientifique, une discussion s'engage entre enseignants à propos des critères éliminatoires]

- Un nul en prépa, c'est pas forcément quelqu'un qui avait 11, ou moins de 11, ça peut être quelqu'un qui avait bien plus que ça en terminale! C'est difficile de connaître les niveaux réels des élèves, ce que signifie un $11 \ldots$

- Un élève qui a 11 mais qui est au taquet, dont ses profs disent qu’il est " très laborieux, très sérieux », ça sert à rien de le prendre!

${ }^{6}$ Valorisation de la « somme de vertus moyennes », qui naît de la tension entre la « dépréciation scolaire du "scolaire" et la nécessaire reconnaissance des vertus proprement scolaires » (Bourdieu, 1989). 
- [Avec de la colère dans la voix] donc il faut prendre quelqu'un qui est pas sérieux, qui est laxiste, qui fout rien!? On ne peut pas pénaliser le gars qui est sérieux quand même, c'est pas possible! Un gars qui a 11 et qui est sérieux, tu vas prendre l'autre qui a 11 mais qui est pas sérieux, qui est fumiste! C'est pas possible!»

La discussion s'arrête là, mais elle est implicitement rappelée (par des postures, des intensités de débats, l'emploi de certaines expressions) dès qu'apparaissent, au fil des dossiers, les catégories du sérieux ou du taquet dans les appréciations.

Elle resurgit enfin explicitement, à l'occasion d'une des dernières discussions sur un cas avant la fin de la séance du matin :

"Quand les profs [i.e. les collègues de lycée] insistent à ce point là, " sérieux, sérieux, sérieux », qu'est-ce qu'on fait ? Quand il n'y a que ça, qu'ils insistent à ce point ?? »

Les enseignants sont directement confrontés à la frontière indécise entre le « sérieux » et le «taquet », et au paradoxe pratique constitué par le fait qu'une catégorie qui témoigne de la docilité scolaire soit affectée négativement. Ils réagissent diversement par rapport à cette contradiction: apparaissent ici des luttes de classements plutôt que des catégories immédiatement partagées, ce qui conduit à se poser la question de ce qui peut fonder et produire ces variations.

On peut faire à ce sujet l'hypothèse qu'il existe quelque chose comme un « espace des styles d'évaluations », dans lequel les classeurs sont classés par leurs classements, et qui, comme l'espace des styles de vie, permet de rendre compte à la fois des jugements, et des jugements sur des jugements, qui sont portés (Bourdieu, 1979). Sans prétendre ici dresser ne serait-ce que les grandes lignes de cet espace, on voudrait juste interroger à cette lumière les rapports des enseignants interrogés à un couple catégoriel, au sein duquel s'inscrit le débat récurrent mentionné ci-dessus sur le « sérieux », et qui se présente pour une large part comme une alternative : le travail ou le niveau comme critère de l'excellence scolaire. Dans quelles 
mesures les propriétés des enseignants permettent-elles d'expliquer leurs prises de position sur cette question?

Il est probable que les variations d'un enseignant à l'autre, dans cet espace des styles d'évaluation, s'organisent selon diverses propriétés : leur âge, génération et ancienneté, le fait d'être ou non soi-même passé par les classes préparatoires et le rapport à ce moment-là de la trajectoire scolaire, le genre (et notamment les conceptions de l'enfance ou de l'école qui y sont attachées), l'origine sociale enfin et le rapport à la culture libre et à la culture scolaire qu'elle peut engendrer. L'enquête n'ayant pas porté sur les trajectoires enseignantes, on ne dispose pas ici de tous les éléments qui permettraient de rendre compte de manière exhaustive de ces propriétés et de leurs effets sur les conceptions enseignantes. En revanche, on peut faire apparaître l'effet de l'une des variables enseignantes, celle de la matière enseignée et surtout de la position dans les hiérarchies enseignantes et dans la division du travail professoral qu'elle entraîne et cristallise. Bien que d'apparence très réductrice par rapport à la prise en compte des multiples autres variables sans doute pertinentes à faire intervenir, elle n'en permet pas moins à elle seule, on va le voir, d'expliquer la distribution des discours entre « travail » et « niveau ».

Lors des entretiens réalisés avec les enseignants, j'ai systématiquement posé la question de ce qu'était, «pour eux », un bon ou un mauvais élève de classes préparatoires ${ }^{7}$. Les réponses à cette question permettent d'accéder à des positionnements explicites sur la question du sérieux, qu'on peut rapporter aux positions du locuteur (notamment dans la hiérarchie des disciplines) ainsi qu'à celles de la classe où il enseigne.

L'examen des entretiens suggère que le fait de mettre en avant le «niveau » des élèves est corrélé avec une position élevée dans l'équipe (matière importante) ou dans les hiérarchies

7 Pour une analyse de cette question et des réponses qu'elle entraîne, et notamment la conclusion selon laquelle "ce qui structure le mieux les attitudes des professeurs en matière de jugement porté sur les élèves, [ce sont] les variables définissant la situation scolaire et sociale de l'enseignant », voir (Léger, 1983). 
symboliques (filières et voies du lycée les plus cotées), alors que les enseignants qui mettent en avant le travail ou la docilité parmi les critères légitimes du bon élève de classes préparatoires se trouvent dans les positions moins élevées («petites matières », classes moins cotées). Le faible nombre d'entretiens réalisés avec des enseignants ne permet pas une généralisation, mais il faut noter que la répartition des enseignants en fonction de ces critères s'établit selon un continuum régulier qui va des enseignants de matières importantes dans les classes les plus cotées (extraits 1) aux enseignants de «petites matières » dans les classes moins cotées (extraits 3), en passant par des positions intermédiaires, occupées en l'occurrence de manière homogène par des enseignants qui enseignent, dans les classes les moins cotées, des matières définies comme «moins importantes » mais qui ne sont pas des «petites matières » (extraits 2 ). Les extraits 1 rassemblent en fait des entretiens où bons et mauvais élèves sont définis par une dimension cognitive du niveau (logique, réflexion, intuition); les extraits 3 tendent à définir bons et mauvais élèves sur une échelle plus personnelle et plus morale, qui implique de ce fait davantage le travail, la docilité ou le fait de savoir suivre les «règles du jeu »; les extraits 2 regroupent des positions intermédiaires, qui mêlent le travail et le niveau, ou encore le cognitif et le comportemental, dans la définition du bon et du mauvais élève.

\section{Encadré $2:$ Le travail ou le niveau?}

Du point de vue des réputations locales et des réussites variables attribuées aux différentes classes (voir encadré 1), il est possible de hiérarchiser les quatre classes observées, en les classant de celle qui est reconnue comme la «meilleure» du lycée, où se trouvent les «meilleurs élèves » qui « ont les meilleurs écoles » (classe 1), à celle dont les enseignants disent qu'il ne s'agit pas pour eux «de faire intégrer les meilleures écoles », mais que « chaque élève trouve une école à son niveau », et qui définissent leur rôle par différence explicite avec celui de leurs collègues de la classe 1 (classe 4). Il faut noter que la hiérarchie 
qui va de la classe 1 à la classe 4, en passant par les classes 2 et 3, s'est retrouvée de manière redondante et consensuelle tout au long de l'enquête, que ce soit dans les discours enseignants, administratifs ou étudiants, ou d'ailleurs dans les réussites effectives des classes observées, tout comme elle correspond à la position de ces classes dans les différents classements médiatiques ou internet qui la manifestent tout autant qu'ils contribuent à la produire. Les quatre classes sont donc codées des plus cotées ( 1 et 2 ) à celles qui le sont moins (3 et 4), les enseignants étant ceux des «matières importantes» en termes de coefficients et/ou de poids symbolique (MI), des «matières moins importantes » (MMI) ou des «petites matières » $(\mathrm{PM})$ de ces classes.

Extraits 1 : Dimension cognitive du niveau

- «Le bon élève, c'est celui qui a de la logique »

«[Il est en train de dire qu'il est difficile de définir un «bon élève » de prépa d'après son travail en terminale] Il y a des profs qui ont essayé des critères, des critères on en a : ça portera justement plus sur l'acquisition de la logique... » (Enseignant, MI, classe 1).

- «Le bon élève, c'est celui qui se pose des questions »

«Alors les très bons élèves, déjà c'est des gens rapides forcément, forcément rapides, avec une bonne mémoire parce que ça leur permet de prendre de l'avance par rapport aux autres et d'avoir enfin le temps de se poser pour réfléchir de temps en temps. Après c'est ceux qui vont avoir de l'intuition, et qui vont surtout avoir un niveau de réflexion qu'on ne rencontre vraiment pas toujours, qui se posent des questions et qui n'essaient pas juste de résoudre une question (...) Qui quand ils tombent sur quelque chose qui leur semble bizarre, que ce soit juste ou faux, qui s'arrêtent et qui se disent : 'Tiens, là y a un problème'. Et il y en a, tous les ans il y en a ici quelques uns » (Enseignant, MI, Classe 2).

Extraits 2 : Positions intermédiaires

- «Le bon élève, c'est celui qui est à l'aise et qui travaille » 
«Celui qui va être le plus à l'aise, c'est déjà celui qui va pas être mis en difficulté intellectuellement, il va tout de suite se sentir bien dans la prépa. Il a compris tout de suite ce qu'on lui demandait, ce que c'était que réfléchir par soi-même (...). Je pense que l'élève travailleur aussi, il ne sera peut-être pas que travailleur, il fera peut-être pas la course en tête, mais il s'en sortira... Bon, mais c'est aussi une valeur, en tant qu'enseignant, qu'on a envie de faire passer : la réussite par le travail... On est peut-être des dinosaures par rapport à ça [rires] ! »(Enseignant, MMI, classes 3 et 4).

- «Le bon élève, c'est celui qui pose des questions »

«Pour moi le bon critère, le critère absolu [du bon élève] - c'est pas suffisant mais presque - c'est l'élève qui pose des questions... Et inversement, s'ils ne vous posent jamais de questions, ça veut dire qu'ils ne s'en posent pas (...). Le trait le plus pertinent chez un bon élève, c'est la curiosité intellectuelle et une forme d'inquiétude intellectuelle, le fait de chercher à comprendre, comment se fait-il que c'est comme ci et pas comme ça... ?»(MMI, classes 3 et 4 )

Extraits 3 : Dimensions morales, personnelles, comportementales.

- «Le bon élève, c'est celui qui rentabilise ce qu'il est »

«Nous on laisse passer des gens qui ont un mauvais classement si on les sent très motivés, travailleurs, susceptibles d'intégrer une petite École...(...) Le bon élève, c'est celui qui rentabilise bien ce qu'il est, et le mauvais, c'est celui qui tire pas profit de ce qu'il est... (...) En tout cas, c'est pas une question de niveau, parce que très clairement à ce niveau là, on voit spontanément qu'il y en a qui sont plus doués que d'autres... Certains sont très laborieux, quand ils décrochent une petite École c'est un miracle, et ça nous réjouit. Donc c'est pas une question de niveau, très clairement (...) Par contre, il y a le bon élève et le mauvais élève au niveau de la classe, par rapport aux autres : le bon élève, c'est celui qui est un élément moteur, et un ciment, et le mauvais élève, c'est celui qui est évaporé, et qui 
empêche les autres de se concentrer, qui va discuter un peu dès que c'est possible » (Enseignant, PM, classes 3 et 4).

- «Le bon élève, c'est celui qui a une bonne santé physique et morale »

«Un bon élève de prépa, c'est un élève qui a une bonne santé physique, déjà pour commencer tout bêtement (...) Et des qualités morales, fermes. L'intellect j'allais dire c'est accessoire : la sélection sur dossier fait que, normalement, on n'a pas de mauvais élèves devant soi. (...) Il y a une nouvelle hiérarchie qui se fonde, qui n'est plus forcément fondée sur des capacités intellectuelles supposées, mais qui est fondée également sur des qualités physiques et, il faut bien le dire, morales. [Comme] Anne [une élève qui est passé en colle la veille, colle à laquelle l'enquêtrice a assisté], le fait de se dire : "Ce que je fais c'est nul, mais tant pis je continue » (...) Le courage oui, tout simplement. Ça ça joue beaucoup. Pour moi, c'est un critère intéressant en classe préparatoire ». (Enseignant, PM, classe 1).

- «Le bon élève, c'est celui qui joue le jeu »

«Moi je considère que [le bon élève] c'est quelqu'un qui est modeste, c'est-à-dire qu'il va se rendre compte qu'il y a une exigence de reprendre ses bases de manière rigoureuse, même s'il se débrouille assez bien, qui va être capable d'apprendre des choses [et qui] a compris que c'est une espèce de règle du jeu un peu particulière (...) Le bon élève finalement, c'est quelqu'un qui va maîtriser ça, qui va être capable de jouer le jeu (...) Le mauvais élève, c'est quelqu'un qui maîtrise pas ses bases, qui a pas envie de les remettre à niveau, qui pense pouvoir se débrouiller tout seul : qui ne joue pas le jeu en fait » (Enseignant, PM, classes 1 et 2).

Ces variations ne sont donc pas seulement des différences de catégorisation : ce sont également des hiérarchies, au sens où les critères de classements du bon et du mauvais élève témoignent de la position des classeurs. On comprend mieux, à cette lumière, les débats même 
larvés qui peuvent apparaître au moment des commissions, ou encore dans les conseils de classes, et les différences qui se font jour en entretien: les catégories de l'entendement professoral, qui jouent fréquemment voire explicitement le rôle d'une langue commune et d'un monde partagé, sont également sujettes à variations, et s'inscrivent dans un espace où elles sont objets de luttes.

\subsection{Prédire : les catégories de la divination professorale}

Si elles ne sont pas uniformes, ces catégories ne sont pas non plus, contrairement à la manière dont elles ont été abordées jusqu'à maintenant, purement évaluatrices. À une opération de sélection, visant à élire les meilleurs élèves de terminale, se superpose en effet une opération de recrutement, visant à identifier les futurs meilleurs élèves possibles en classes préparatoires, ce qui s'avère n'être pas la même chose. De ce point de vue, la sélection des dossiers s'apparente à une opération de «prédiction » sociale et scolaire ${ }^{8}$.

Discerner les prédispositions à être un bon élève de classe préparatoire se fait dans un contexte d'incertitude relative, distinct de l'assurance que pourrait donner une pure évaluation des qualités manifestées par les dossiers scolaires. Lors des commissions, certaines remarques peuvent faire penser que ce sont des certitudes qui président à l'opération de sélection, mais cette attitude confiante est avant tout une attitude fataliste, et ne concerne que les dossiers dont les enseignants pensent que leur possesseur court de très grands risques d'avoir des difficultés en classe préparatoire. Une telle attitude est visible dans des formules du type : «En le/la prenant, on le/la condamne à [avoir 2/20 toute l'année, ramer, jamais décoller en telle matière, être en quеue de classement, etc.] » qui parsèment les propos sur les dossiers problématiques, ou encore dans les anticipations de notes, voire de «bulletins » qui sont énoncées : [Commission d'admission en prépa commerciale. On est dans le premier tiers des

${ }^{8}$ L'enjeu « prédictif » est également central dans les opérations de sélection et de recrutement dans les grandes universités américaines analysées sur près d'un siècle par (Soares, 2007) et (Karabel, 2006). 
dossiers, donc a priori ceux qui sont gardés. Dossier d'une élève, bonne moyenne mais pas en maths]. Un enseignant lit tout haut : "En maths, elle a 'le travail n'est pas à la hauteur des besoins.'... ça ça veut dire 1 [1/20 en maths] en prépa! (...) Le bulletin de l'année prochaine est déjà prêt : 'des lacunes à combler'” !».

Mais si les cas problématiques peuvent faire consensus dans les prédictions qui sont faites à leur propos, il ne s'agit que d'exceptions à une situation générale marquée par l'incertitude : " [Commission d'admission en prépa commerciale. On est dans le troisième tiers des dossiers]. À propos d'un dossier, un enseignant demande 'Que vaut un 14 en maths dans une classe de niveau 4?", à quoi un autre répond: "je ne sais pas si un 14 en terminale ça veut dire quelque chose de toute façon!' [A propos d'un autre dossier :] 'Une mauvaise moyenne en histoire est un signe inquiétant, une très bonne moyenne est un signe de rien du tout!"”.

Ainsi donc, il s'agit bien de rechercher des «signes » d'excellence ou de qualité ultérieures, et ces derniers ne sont pas faciles à lire ${ }^{9}$. Les deux exemples ci-dessus étant extraits des procédures de sélection pour les classes préparatoires commerciales, on pourrait penser que la certitude est davantage l'apanage des commissions scientifiques, ou des enseignants de sciences. Mais ils expriment également l'incertitude prédictive de la lecture des signes scolaires, notamment en la rapportant au schème travail/niveau déjà identifié plus haut comme une catégorie centrale de la perception préparatoire :

«Je sais pas quel est le bon profil d'élève de terminale (...) C'est sur dossier, donc on peut pas connaître le profil, bon là on a essayé de cibler le profil, et on n'y est pas [arrivé]. Enfin moi j'ai toujours pas trouvé le bon profil, parce que, un élève qui travaillait déjà beaucoup, la question est : 'est-ce qu'il avait besoin de travailler beaucoup pour atteindre ce

9 A la différence, semble-t-il, de "l'interprétation des signes de l'appel » et de l'usage des critères de sélection (comportementaux, ou de «valeur de l'enfant ») dans les procédures de sélection des petits séminaristes (Suaud, 1978). 
niveau-là, ou est-ce qu'il travaillait beaucoup parce que c'est sa nature? '. Alors, l'élève qui travaille beaucoup parce que c'est sa nature, et qu'était très bon, celui-là, ça sera un excellent élève de prépa. Un élève qui travaillait beaucoup, parce qu'il en avait besoin pour atteindre ça, ça sera en général un élève médiocre : malheureusement, en classe prépa, il arrivera pas à soutenir le rythme, s'il a déjà besoin de travailler tout ça en terminale, en classe prépa il soutiendra pas le rythme. Après, on a de l'autre côté les élèves qui travaillent peu en terminale, parce que finalement on a été peu exigeant vis-à-vis d'eux, puis arrivés en prépa, de manière naturelle, on devient plus exigeant, ils se mettent au travail, pfff...[ils s'envolent]. Finalement ceux-là vont être excellents. Puis ceux qui vont avoir du mal à se mettre au travail, ceux-là vont être moyens. Et puis ceux qui s'y mettront pas, ceux-là vont être «mauvais» entre guillemets. Donc finalement, leur attitude par rapport au travail en terminale n'est pas un bon critère, c'est pas un critère qui permet de discriminer la population de manière intéressante, même ceux qui travaillaient, c'est ça qui est embêtant, à la limite, on pourrait dire, ceux qui travaillent pas, on les prend, on verra bien, et ceux qui travaillent, on les prend parce que c'est sûr, et ben non, ça marche pas... »(Enseignant, science, prépa scientifique).

Comme le montre cet extrait, l'incertitude qui préside au recrutement sur dossiers, ou plus exactement l'incertitude sur le devenir en classes préparatoires de tel ou tel élève de lycée est liée de manière fondamentale à la caractérisation du bon élève de classes préparatoires comme un ancien lycéen qui en avait «sous la pédale » et qui va même en garder «sous la pédale » jusqu'en deuxième année (l'expression revient très souvent dans les conseils de classe de première année), à l'inverse de l'excellent élève de lycée qui a en fait «tout donné » pour parvenir à ce statut : «Certains travaillent trop oui, surtout en première année. C'est la même logique qu'au Bac. Vous avez au Bac des élèves qui obtiennent le Bac avec une mention Très Bien, mais comme on dit ils sont carbonisés, ils sont à bout. Ils vont 
arriver en classe prépa, on demande de passer à la vitesse supérieure et ils ne peuvent plus... Et on a en première année quelques élèves qui sont comme ça » (Enseignant, lettres, prépa commerciale). Les signes du travail et du niveau sont donc intrinsèquement réversibles d'un point de vue prédictif : le travail peut être la marque positive de la capacité de travail, ou celle, négative, du besoin d'un travail important en terminale. Le bon niveau peut être le signe positif des compétences scolaires et intellectuelles ou le stigmate négatif d'un investissement disproportionné par rapport à l'enjeu de la terminale, et de ce fait brûlé ou gaspillé pour la suite.

Ces cas d'incertitude pronostique permettent de voir ce qui constitue, au fond, le cœur de ces opérations de divination professorale : la quête, toujours incertaine, de ce qu'on pourrait appeler «l'énergie scolaire»d'un candidat. Si l'on emprunte ce terme d'énergie, et sa définition duale en termes de «capacité » et d' «obéissance», à M. Foucault ${ }^{10}$, c'est précisément parce qu'il permet de concentrer les deux réquisits, scolaire et comportemental, que les classes préparatoires exigent simultanément des candidats : la quête professorale s'inscrit dans un «art du corps humain qui ne vise pas seulement la croissance de ses habiletés, ni non plus l'alourdissement de sa sujétion, mais la formation d'un rapport qui dans le même mécanisme le rend d'autant plus obéissant qu'il est plus utile, et inversement » (Foucault, 1975). Comme les enseignants ne disposent pas, dans la plupart des classes préparatoires, de moyen de se représenter le collectif auquel aboutira in fine l'opération de sélection qu'ils ont menée (sauf peut-être tout en haut de la hiérarchie préparatoire), cette recherche de l'énergie individuelle est la seule qui permette d'agir sur l'énergie du collectif

10 « La discipline majore les forces du corps (en termes économiques d'utilité) et diminue ces mêmes forces (en termes politiques d'obéissance). D'un mot : elle dissocie le pouvoir du corps ; elle en fait une "aptitude", une "capacité" qu'elle cherche à augmenter; et elle inverse d'autre part l'énergie, la puissance qui pourrait en résulter, et elle en fait un rapport de sujétion stricte (...) La coercition disciplinaire établit dans le corps le lien contraignant entre une aptitude majorée et une domination accrue » (Foucault, 1975), je souligne. 
qui composera la classe de première année ${ }^{11}$. Cette énergie doit répondre à des caractéristiques précises : elle doit être présente chez l'élève (c'est-à-dire perceptible dans son dossier), mais davantage sous une forme latente que mobilisée, l'idéal étant que le dossier porte les signes de son accumulation et de sa conservation, de son absence de dissipation par l'élève. Les indices de sa surexploitation, dès le lycée, sont en tout cas aussi néfastes que ceux de son absence. Ainsi, le fait que les résultats d'un élève aient baissé entre le premier et le deuxième trimestre de terminale est un signe très mauvais, le fait qu'ils aient augmenté sera en revanche évalué très positivement. La recherche de ce type d'indice manifeste donc bien que ce qui est recherché est moins un niveau qu'un potentiel, moins un état qu'une énergie, moins des dispositions déjà présentes que des dispositions à développer ultérieurement d'autres dispositions.

La conception des entrants en classes préparatoires comme gisement collectif d'énergie scolaire est en fait cohérente avec un enjeu et une fonction centrale de la classe préparatoire telle qu'elle apparait dans l'enquête : il s'agit d'un dispositif destiné à mettre une population au travail. On pourrait penser en effet que sélection des entrants et fonctionnement de la classe préparatoire comme dispositif de mise au travail sont antinomiques, ou au mieux redondants. L'institution, par la lourdeur de ses procédures de sélection, élirait si bien ses membres qu'elle n'aurait plus qu'à se reposer sur leurs dispositions et prédispositions, sans avoir elle-même de travail particulier à fournir pour obtenir leur investissement, et ce d'autant plus qu'elle peut compter sur le fait que les élèves partagent les objectifs finaux qui leurs sont assignés (avoir une ou telle école, et la « meilleure » possible). Or la sélection analysée ici est

11 À la différence sur ce point des responsables des admissions dans le Collège étudié par M. Steven (2007), qui recrutent une "promotion» ou une "cohorte" - alors que les entraîneurs sportifs, qui participent également à la procédure aux États-Unis, élisent certains candidats athlètes sur une base individuelle. 
bien plutôt le premier et non le dernier des mécanismes sur lesquels s'appuie l'institution pour s'assurer la coopération et l'investissement des élèves. De là le fait que les enseignants cherchent paradoxalement moins de «bons élèves» de lycée que des élèves qui vont justement pouvoir être mis au travail, qui en ont «sous la pédale» et qui ne sont pas «carbonisés ». D'où aussi l'incertitude relative dans laquelle s'inscrit cette quête, pour laquelle le dossier scolaire ne livre que des indications ambiguës.

La classe préparatoire fait en effet partie de ces institutions qui redoublent une opération de recrutement (et d'auto-sélection) minutieuse et ciblée sur des dispositions situées très précisément dans l'espace social, par une activité visant à assurer un contrôle et une influence institutionnels maximaux sur les membres ainsi sélectionnés. Comparable en cela au petit séminaire (Suaud, 1978), à l'armée (Pinto, 1975) ou au gym (Wacquant, 2000), l'activité institutionnelle ne s'épuise ni dans son travail de sélection ni dans son travail de transformation du «matériau humain »(Goffman, 1968) sur lequel elle opère, mais les combine successivement. C'est à cette lumière qu'il faut interpréter les opérations analysées ici : elles constituent une procédure visant à sélectionner une population à mettre au travail. Même quand on l'a soigneusement pêché, « enseigner au poisson à nager » (Bourdieu, 1989) ne s'avère finalement pas si simple.

Muriel Darmon

CNRS, Centre Max Weber

ENS de Lyon

Muriel.Darmon@ens-lyon.fr

\section{Références bibliographiques}

ABRAHAM Y.-M. 2007 Du souci scolaire au sérieux managérial, ou comment devenir un « HEC ». Revue Française de Sociologie, vol. 1, n 48, p. 37-66.

ALLOUCH A., VAN ZANTEN A. 2008 Formateurs ou «grands frères »? Les tuteurs des programmes d'ouverture sociale des Grandes Écoles et des classes préparatoires. Éducation et Sociétés, vol. 1, n 21, p. 49-65. 
BOURDIEU P. 1979 La Distinction. Paris : Minuit.

BOURDIEU P. 1989 La Noblesse d'État. Paris : Minuit.

BOURDIEU P. 1993 Actualité et fécondité de l'œuvre de Durkheim en sociologie de l'éducation, in Cardi F., Plantier J. (dir.). Durkheim. Sociologue de l'éducation. Paris : L'Harmattan.

BOURDIEU P., PASSERON J.-C. 1985 Les Héritiers. Paris : Minuit.

BOURDIEU P., SAINT MARTIN M. de 1975 Les catégories de l'entendement professoral. Actes de la Recherche en Sciences Sociales, vol. 1, n 3, p. 68-93.

FOUCAULT M. 1975 Surveiller et punir. Paris : Gallimard.

GOFFMAN E. 1968 [1961] Asiles. Paris : Minuit.

KARABEL J. 2006 The Chosen. The Hidden History of Admission and Exclusion at Harvard, Yale, and Princeton. New York, NY : Mariner Books.

LAGROYE J. 1997 Sociologie politique. Paris : PFNSP et Dalloz.

LÉGER A. 1983 Enseignants du secondaire. Paris : PUF.

MARRY C. 2000 Filles et garçons à l'école, in Van Zanten A. (dir.). L'École. L'état des savoirs. Paris : La Découverte, p. 283-292.

MARRY C. 2004 Les Femmes ingénieurs. Une révolution respectueuse. Paris : Belin.

MERLE P. 1998 Sociologie de l'évaluation scolaire. Paris : PUF, Paris.

PASQUALI P. 2010 Les déplacés de «l'ouverture sociale». Sociologie d'une expérimentation scolaire. Actes de la Recherche en Sciences Sociales, vol. 3, n 183, p. 86103.

PINTO L. 1975 L'armée, le contingent et les classes sociales. Actes de la Recherche en Sciences Sociales, vol. 1, n ${ }^{\circ}$ 3, p. 18-40.

PONS A. 2007 Les étudiants en classes préparatoires aux grandes écoles. Année 20062007. Note d'information 07-37 MEN-DEPP.

SAINT MARTIN M. de 2008 Les recherches sociologiques sur les grandes écoles : de la reproduction à la recherche de justice. Éducation et Sociétés, vol. 1, n 21, p. 95-103.

SOARES J. A. 2007 The Power of Privilege. Yale and America's Elite Colleges. Stanford : Stanford University Press.

STEVENS M. L. 2007 Creating a Class. College Admissions and the Education of Elites. Cambridge, London : Harvard University Press.

SUAUD Ch. 1978 La Vocation. Paris : Minuit.

WACQUANT L. 2000 Corps et âme. Marseille : Agone. 